\title{
Les techniques de dosimétrie passive
}

\author{
A. BIAU
}

La dosimétrie passive repose sur l'utilisation de dosimètres qui intègrent des doses sur une période donnée, mois ou trimestre, et sont traités selon des procédés physicochimiques spécifiques dans des laboratoires spécialisés.

La dosimétrie active, pour sa part, donne en temps réel les doses et débits de dose directement accessibles au porteur de ce type de dosimètre.

Historiquement, les premiers dosimètres reposaient sur la photographie (réduction d'ions d'argent sous l'effet des rayons $\mathrm{X}$ ou $ү$ ) et l'ionisation des gaz, compteurs, chambre d'ionisation.

Plus récemment, à la fin des années 1960, sont apparues la radio-thermoluminescence (TLD) et la radio-photo-luminescence (RPL) et, dans les années 1990, la luminescence optiquement stimulée (OSL).

\subsection{La dosimétrie photographique}

Les découvertes de la photographie et des rayonnements ionisants, $X$ et $\gamma$ sont contemporaines à la fin du $\mathrm{XIX}^{\mathrm{e}}$ siècle et début du $\mathrm{XX}^{\mathrm{e}}$. Les découvertes d'Henri Becquerel, Pierre et Marie Curie ou Ernest Rutherford ont été mises en évidence souvent à partir de supports photographiques. La figure 2.1 ci-dessous montre la photo de William Conrad Roentgen et la radiographie qu'il pratiqua sur la main de son épouse Bertha Roentgen (cliché en positif).
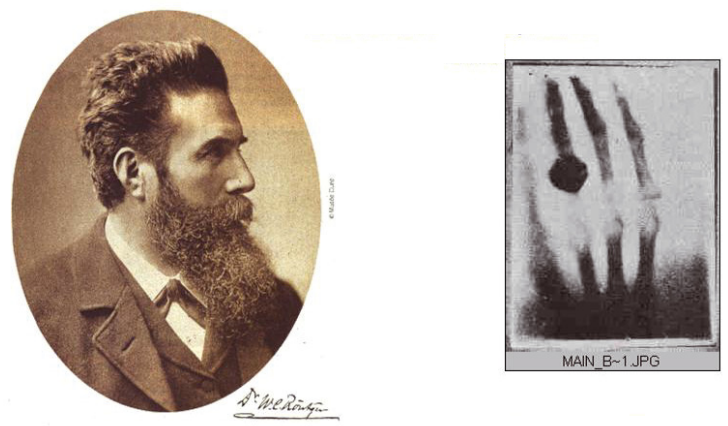

Figure 2.1 - Découverte des rayons X en 1895. À gauche, portrait de William Conrad Roentgen ; à droite, première radiographie de la main de son épouse, Bertha Roentgen. 
Il est donc logique que pour détecter la présence de photons et en évaluer la quantité on ait fait appel à la photographie.

L'émulsion photographique a été fixée sur différents supports comme le verre pour les premières radiographies, puis sur un film en acétate de cellulose ou le papier. Dans les premiers temps de l'utilisation des rayons $\mathrm{X}$ en radiologie médicale ou au début de l'aventure du nucléaire militaire et civil, il y a eu des dosimétries sommaires avec des films de type dentaire sans écran qui étaient extrêmement imprécis mais qui pouvait attester de la présence de rayonnements. Les dosimètres photographiques se sont perfectionnés avec l'utilisation de badges comportant des écrans de matériaux divers permettant d'apprécier l'énergie des photons et améliorer la conversion du noircissement en dose.

Les principaux fabricants de dosimètre photographique ont été les grands industriels de la photographie, Kodak, Agfa, Dupont de Nemours, Fuji, Illford.

En France, les différents laboratoires ont utilisé les dosimètres sur support papier de Kodak France selon un modèle créé par Norbert Chassende-Baroz (figure 2.2) et ensuite les dosimètres films de Kodak France et US et Agfa.

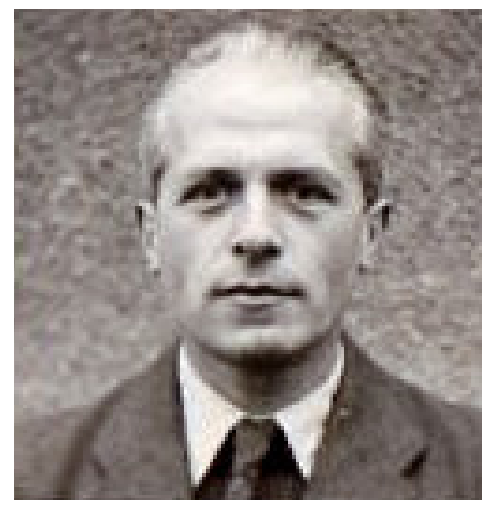

Figure 2.2 - Norbert Chassende-Baroz.

\subsubsection{Le dosimètre photographique papier}

Le dosimètre photographique sur support papier de type Chassende-Baroz a été utilisé dans la plupart des laboratoires français jusqu'à la fin des années 1970. Ce dosimètre (figure 2.3) comportait un support en carton de $3 \times 4 \mathrm{~cm}$ sur lequel étaient collées, d'un côté une bande automarquante pour identifier le dosimètre et deux bandes de papier photographique et sur l'autre face deux autres bandes de papier photographique dont une comme témoin de développement. Le tout était placé dans un conditionnement étanche à la lumière et à l'humidité. 

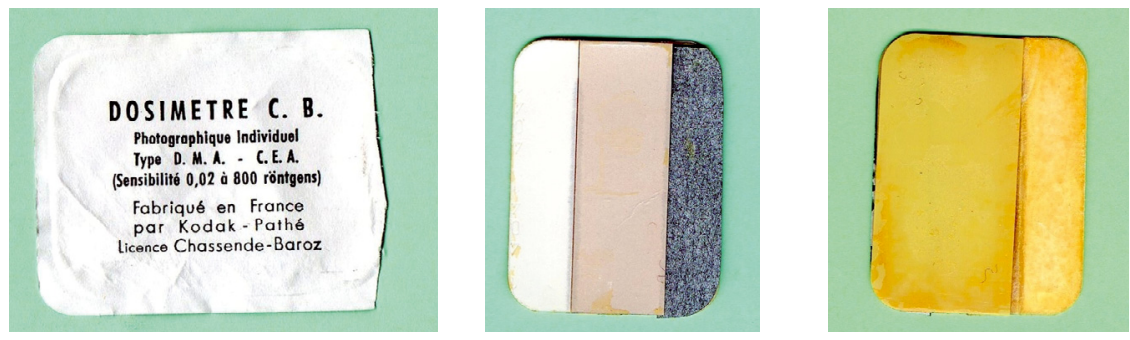

Figure 2.3 - Dosimètre photographique Chassende-Baroz.

L'image de gauche représente le dos de la pochette du dosimètre, au centre les deux émulsions et la plage de marquage, à droite, la troisième émulsion et le témoin de développement.

Il y avait donc trois émulsions de sensibilité différentes permettant de couvrir une grande plage de doses. En effet, si on utilise une seule émulsion très sensible elle est vite saturée et on ne peut pas mesurer les doses supérieures et si elle est peu sensible, le seuil de mesure est trop élevé.

Ce dosimètre était ensuite placé dans un support contenant des écrans comme le plomb ou le cuivre et l'ensemble dans une pochette plastique avec un dispositif d'attache pour le fixer sur le vêtement du porteur.

La nature des écrans et les protocoles de traitement de ces dosimètres ainsi que les modes de calibration étaient variables selon les laboratoires comme nous le verrons aux chapitres $6,7,8,9,10$ et 11 .

Une fois développés et fixés, ces dosimètres présentaient des noircissements ou densités optiques qu'il fallait mesurer pour déterminer la dose reçue. Ces mesures étaient effectuées avec des densitomètres à réflexion. Un faisceau lumineux d'intensité de référence était projeté sur les différentes parties du dosimètre et on mesurait l'intensité de lumière réfléchie, le pourcentage de lumière réfléchie étant lié au noircissement et donc à la dose reçue par le dosimètre.

\subsubsection{Le dosimètre photographique film («dosifilm »)}

Ce type de dosimètre, que l'on a rapidement appelé « dosifilm », est apparu dès le début des années 1970 et cette dénomination est encore utilisée alors qu'il n'y a plus en France de tels dosimètres depuis le début des années 2000 !

Il y eut d'abord des dosimètres comprenant dans la même enveloppe deux supports sous forme de film, l'un recouvert d'une émulsion sensible (« rapide») 
pour mesurer les doses faibles et l'autre avec une émulsion moins sensible («lente ») pour les doses plus élevées.

Ensuite ce dosimètre était, comme le dosimètre papier, placé dans un étui contenant des filtres et une enveloppe plastique. Le mode de traitement appartenait au laboratoire qui les fournissait.

Ces dosimètres Kodak type 3 et Agfa ont été utilisés surtout dans un premier temps pour des raisons économiques, le dosimètre papier fabriqué à Vincennes par Kodak France étant nettement plus cher.

Cependant le fait qu'il y ait deux films par dosimètre est devenu un obstacle pour les laboratoires les plus grands car, au cours des années 1970, le nombre de travailleurs surveillés a crû très rapidement en raison notamment de l'essor du nucléaire civil.

Vers 1975 est apparu le dosimètre Kodak type 2 qui avait la particularité de présenter les deux émulsions comme le Kodak type 3 décrit sur la figure 2.4 mais sur un seul film, avec l'émulsion rapide sur une face et l'émulsion lente sur l'autre (schéma ci-dessous). Le gros avantage était qu'il y avait deux fois moins de films à développer mais l'inconvénient était que lorsque l'émulsion rapide était saturée, à partir de $3 \mathrm{mSv}$, il fallait l'enlever par «grattage » pour pouvoir lire l'émulsion lente sur l'autre face. Heureusement cette opération n'était nécessaire que pour des doses élevées qui n'étaient pas très fréquentes (environ $10 \%$ des dosimètres).

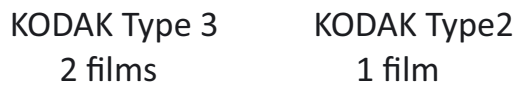

1 film

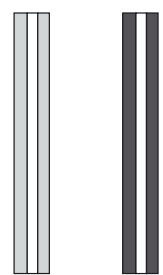

\section{Emulsion lente Emulsion rapide}

Figure 2.4 - Constitution des dosimètres KODAK Type 3 et Type 2.

Les mesures sur ces dosimètres films étaient effectuées avec des densitomètres à transmission, un faisceau lumineux d'intensité $I_{0}$ était projeté sur le dosimètre et la mesure de l'intensité transmise $I$ permettait de déterminer la densité optique $d$ liée à la dose reçue par le dosimètre :

$$
d=\log \left(I_{0} / I\right)
$$



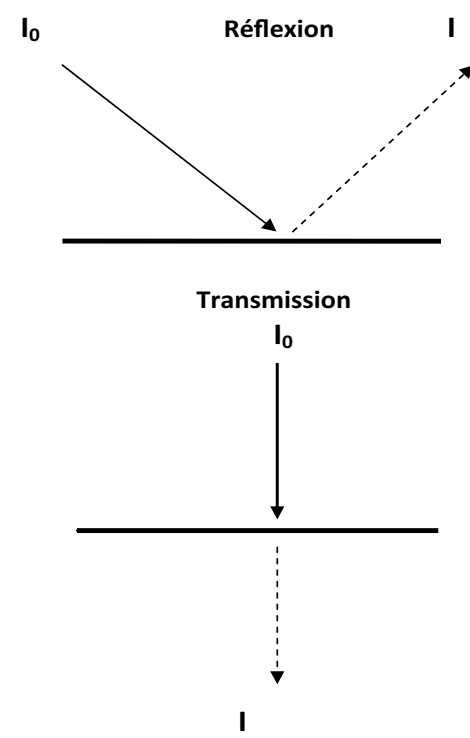

Dosimètre papier (Kodak T1)

Dosimètre film (Agfa, KodakT2 et T3)

Figure 2.5 - Principe de la lecture des dosimètres photographiques par réflexion et transmission.

Les principes de mesure de la densité optique par réflexion et transmission sont représentés sous forme de schémas sur la figure 2.5 .

Le tableau I montre la répartition des dosimètres photographiques par catégorie et par laboratoire au fil du temps entre 1973 et 2008.

TABLEAU I

Les dosimètres photographiques utilisés dans les principaux laboratoires de dosimétrie photographique individuelle de 1973 à 2008.

\begin{tabular}{lccccc}
\hline Année & SCPRI/OPRI/IRSN & CEA/IPSN & Défense & LCIE & COGEMA/AREVA \\
\hline 1973 & KODAK T3 /AGFA & KODAK CB & KODAK CB & KODAK CB & KODAK T3 \\
1974 & KODAK T3/AGFA & KODAK CB & KODAK CB & KODAK CB & KODAK T3 \\
1974 & KODAK T3/AGFA & KODAK CB & KODAK CB & KODAK CB & KODAK T3 \\
1975 & KODAK T3/AGFA & KODAK CB & KODAK CB & KODAK CB & KODAK T3 \\
\hline 1976 & KODAK T2 & KODAK CB & KODAK CB & KODAK CB & KODAK T3 \\
1977 & KODAK T2 & KODAK CB & KODAK CB & KODAK CB & KODAK T3 \\
\hline 1978 & KODAK T2 & KODAK CB & KODAK CB & KODAK CB & KODAK T3 \\
1979 & KODAK T2 & KODAK CB & KODAK CB & KODAK CB & KODAK T3 \\
\hline
\end{tabular}


TABLEAU I

Suite.

\begin{tabular}{|c|c|c|c|c|c|}
\hline Année & SCPRI/OPRI/IRSN & CEA/IPSN & Défense & LCIE & COGEMA/AREVA \\
\hline 1980 & KODAK T2 & KODAK CB & KODAK CB & KODAK CB & KODAK T3 \\
\hline 1981 & KODAK T2 & KODAK CB & KODAK CB & KODAK CB & KODAK T3 \\
\hline 1982 & KODAK T2 & KODAK CB & KODAK CB & KODAK CB & KODAK T3 \\
\hline 1983 & KODAK T2 & KODAK CB & KODAK CB & KODAK CB & KODAK T3 \\
\hline 1984 & KODAK T2 & KODAK CB & KODAK CB & KODAK CB & KODAK T3 \\
\hline 1985 & KODAK T2 & KODAK CB & KODAK CB & KODAK CB & KODAK T3 \\
\hline 1986 & KODAK T2 & KODAK CB & KODAK CB & KODAK CB & KODAK T3 \\
\hline 1987 & KODAK T2 & KODAK CB & KODAK CB & KODAK CB & KODAK T3 \\
\hline 1988 & KODAK T2 & KODAK CB & KODAK CB & KODAK CB & KODAK T3 \\
\hline 1989 & KODAK T2 & KODAK CB & KODAK CB & KODAK CB & KODAK T3 \\
\hline 1990 & KODAK T2 & KODAK CB & KODAK CB & KODAK CB & KODAK T3 \\
\hline 1991 & KODAK T2 & KODAK CB & KODAK CB & KODAK CB & KODZK T3 \\
\hline 1992 & KODAK T2 & KODAK CB & KODAK CB & KODAK CB & KODAK T3 \\
\hline 1993 & KODAK T2 & AGFA & AGFA & KODAK CB & KODAK T3 \\
\hline 1994 & KODAK T2 & AGFA & AGFA & KODAK T2 & KODAK T3 \\
\hline 1995 & KODAK T2 & AGFA & AGFA & KODAK T2 & KODAK T3 \\
\hline 1996 & KODAK T2 & AGFA & AGFA & KODAK T2 & KODAK T3 \\
\hline 1997 & KODAK T2 & AGFA & AGFA & KODAK T2 & KODAK T3 \\
\hline 1998 & KODAK T2 & AGFA & AGFA & KODAK T2 & KODAK T2 \\
\hline 1999 & KODAK T2 & AGFA & AGFA & - & KODAK T2 \\
\hline 2000 & KODAK T2 & AGFA & AGFA & - & KODAK T2 \\
\hline 2001 & KODAK T2 & AGFA & AGFA & - & KODAK T2 \\
\hline 2002 & KODAK T2 & AGFA & AGFA & - & KODAK T2 \\
\hline 2003 & KODAK T2 & - & AGFA & - & - \\
\hline 2004 & KODAK T2 & - & AGFA & - & - \\
\hline 2005 & KODAK T2 & - & - & - & - \\
\hline 2006 & KODAK T2 & - & - & - & - \\
\hline 2007 & KODAK T2 & - & - & - & - \\
\hline 2008 & KODAK T2 & - & - & - & - \\
\hline
\end{tabular}

*CB : Chassende Baroz

\subsubsection{Avantages et inconvénients du dosimètre photographique}

Du point de vue strictement dosimétrique, le dosimètre photographique n'a jamais été le meilleur mais il présentait des avantages en termes économiques et pratiques pour effectuer une surveillance dosimétrique à grande échelle et il faut noter que la plupart des pays ont utilisé le dosimètre photographique. Il faut dire aussi qu'au 
fil du temps les contextes évoluent, les techniques aussi et l'intérêt des unes par rapport aux autres de la même façon.

\section{Principaux avantages}

Le coût : le coût du film lui-même, des produits de traitement sont relativement faibles et le poids permettait d'expédier par la poste deux dosimètres à un cabinet dentaire, par exemple, dans une enveloppe affranchie au tarif « lettre urgente » de base. Même pour un grand hôpital avec 500 dosimètres le prix de l'affranchissement demeurait raisonnable. C'était un atout important pour des laboratoires qui envoyaient chaque mois des dosimètres à plusieurs milliers d'établissements.

La simplicité : le traitement chimique des dosimètres, développement, bain d'arrêt, fixage et séchage est classique et ne présente pas de difficulté particulière sinon celle de maîtriser la température des bains. Le procédé de lecture au moyen de densitomètres est aussi très simple, la seule contrainte est de bien contrôler les dérives électroniques au cours du temps.

La possibilité de relecture : une fois lus et interprétés, les films sont classés et archivés, de sorte que si à réception du résultat le médecin du Travail a des doutes sur ce résultat, il peut faire une enquête avec les principaux intéressés, porteur du dosimètre, personne compétente en radioprotection, employeur, et demander une relecture du dosimètre qui confirmera le résultat, le modifiera ou l'annulera.

L'exploitation d'une image : le dosimètre photographique étant inséré dans un étui porte-écrans, l'exposition aux rayonnements $\mathrm{X}$ ou $\gamma$ fait apparaitre sur ce dosimètre différentes zones avec des densités différentes selon l'atténuation des rayonnements par ces écrans. Les rapports entre ces différentes densités permettent d'évaluer l'énergie du rayonnement en cause et déterminer la dose avec une plus grande précision.

La figure 2.6 ci-dessous montre la disposition des écrans sur le film et des images de dosimètres exposés à des rayons $\mathrm{X}$ et des rayons $\mathrm{\gamma}$.

Sur le film exposé aux rayons $\mathrm{X}$ de $80 \mathrm{kV}$, on observe trois zones bien distinctes, la partie non filtrée présente la densité optique maximale, sous l'écran de cuivre la densité est plus faible et sous le plomb il n'y a pratiquement pas d'exposition.

Sur le film exposé aux rayonnements $\gamma$ du cobalt 60 , l'image est inversée : la partie nue présente la plus faible densité optique car les rayonnements $\gamma$ d'énergie 


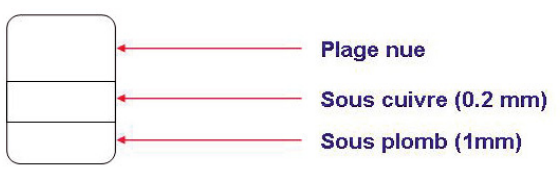

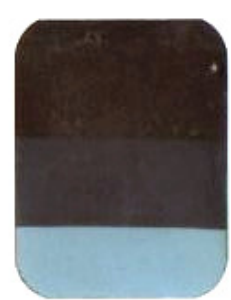

(a)

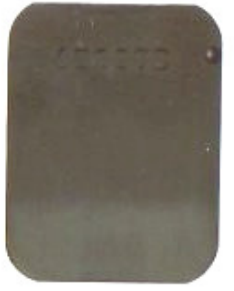

(b)

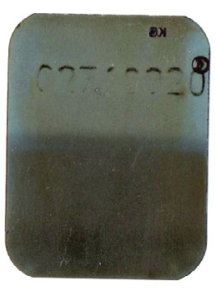

(c)

Figure 2.6 - Dosimètres SCPRI exposés à 1,0 millisievert, (a) rayons X de $80 \mathrm{kV}^{1}$, (b) rayons ү de l'Iridium 192, (c) rayons $\vee$ du cobalt 60.

1173 et $1332 \mathrm{keV}$ traversent le film en ne transférant que peu d'énergie compte tenu de la faible épaisseur émulsion + support, par contre sous les écrans de cuivre et de plomb la densité optique est plus élevée car les photons incidents interagissent avec le métal des écrans et des rayons secondaires sont émis entraînant le noircissement du film.

Le film exposé aux rayons $\gamma$ de 317 et $468 \mathrm{keV}$ de l'iridium 192 présente une image intermédiaire observée fréquemment chez les utilisateurs d'appareils de gammagraphie et parfois chez des médecins de curiethérapie implantant des fils d'iridium à des patients.

C'est l'intérêt de la dosimétrie photographique, mais à cette occasion on peut parfois mettre en évidence certaines circonstances d'exposition non conforme qui peuvent être source d'erreurs : différences de densités correspondant à une énergie de photons différente de celle des rayonnements utilisés habituellement, parfois mélange de rayonnements d'énergies différentes, par exemple en radiothérapie, exposition en $\mathrm{X}$ sur l'installation de centrage du patient et en $\gamma$ lors du traitement de ce même patient. Il y a aussi des cas où une partie seulement du dosimètre est exposée, soit parce qu'un objet s'est interposé entre le dosimètre et la source de rayonnements, stylo, montre, alliance, soit que le champ de rayonnement ne couvrait qu'une partie du dosimètre, champ très localisé en dentaire ou en diffraction X (cristallographie). Voir les images de la figure 2.7.

\footnotetext{
1 Pour les rayons X, il est d'usage de caractériser le spectre énergétique par la haute tension en kilo Volts appliquée aux bornes du tube.
} 


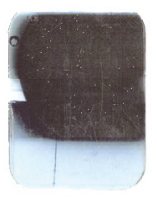

(a)

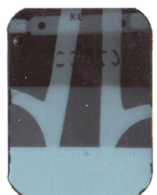

(e)

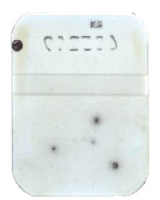

(b)

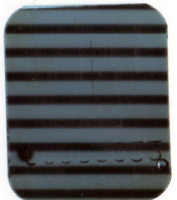

(f)

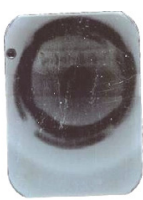

(c)

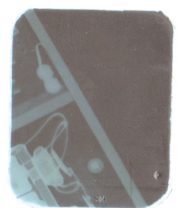

(g)

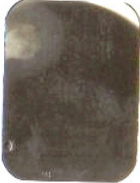

(d)

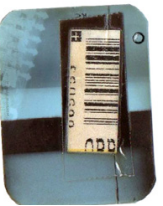

(h)

Figure 2.7 - Images observées sur des dosimètres photographiques du SCPRI / OPRI.

(a) faisceau dentaire, (b) traces de contamination, (c) détecteur de fumée (amércium 241), (d) image du pouce d'un opérateur tenant le dosimètre entre le pouce et l'index, (e) paire de ciseaux, $(f)$ coupes de scanner, (g) objets non identifiés, $(h)$ ressort de stylo.

Le dosimètre «f » a été exposé à l'Hôpital de la Pitié Salpêtrière pour une expertise (Patrice Fraboulet), les autres ont été retournés au laboratoire dans la masse des dosimètres mensuels à traiter sans explication particulière. Les images observées ne sont pas forcément liées à des expositions volontaires et donnent des indications sur les conditions de travail. En particulier le dosimètre (b) présente des traces de contamination dues à une dispersion de radioactivité qui ont peut être entraîné une dose interne pour le porteur du dosimètre.

Les images que l'on observe sur les dosimètres (e), (g) et (h) sont dues au fait que le dosimètre était souvent porté dans la poche poitrine du vêtement de travail dans laquelle pouvait se trouver des objets divers métalliques, le cas le plus fréquent étant le stylo comme sur le film « $\mathrm{h}$ » où l'on observe le ressort métallique. La netteté des images indique que l'exposition a été délivrée en position fixe et exclue une exposition chronique durant la période de port du dosimètre.

Les inhomogénéités observées montrent la nécessité d’observer chaque dosimètre pour faire une lecture correcte et significative de la dose reçue. En effet, comme vu plus haut, la lecture se fait en projetant sur le dosimètre photographique un faisceau lumineux très fin (environ $2 \mathrm{~mm}^{2}$ ) en un, deux ou trois points selon la précision que l'on recherche et par conséquent si le noircissement n'est pas homogène, selon l'endroit de la mesure, le résultat peut être significativement différent. Par exemple, si sur le dosimètre (b) ci-dessus on mesure sur les taches de contamination, la dose sera positive alors que sur le reste du film elle est nulle, 
à l'inverse pour le dosimètre (e) si la mesure est faite sur la partie protégée par les ciseaux la dose sera très largement sous-estimée. Cette possibilité d'exploiter des images constitue un avantage pour élucider certaines circonstances d'exposition mais, en contrepartie, l'automatisation de la lecture en des points identiques sur tous les dosimètres peut entraîner des erreurs conséquentes.

\section{Principaux inconvénients}

La fragilité : pour les dosimètres en conditionnement papier (Kodak types 2 et 3) si le dosimètre n'était pas inclus dans une pochette plastique étanche, l'émulsion pouvait être attaquée par l'humidité, combinée à la chaleur, dans les pays africains ou les DOM TOM par exemple ou par les vapeurs de mercure issues des amalgames dans les cabinets dentaires. Dans les deux cas les dosimètres pouvaient être ininterprétables. La chaleur seule aussi pouvait être à l'origine du décollement du conditionnement et l'entrée de lumière entraînait la destruction du dosimètre.

Les seuils de mesure : l'émulsion photographique est beaucoup plus sensible aux photons de basse énergie inférieure à $100 \mathrm{keV}$ qu'aux photons d'énergie supérieure à $400 \mathrm{keV}$ comme ceux de l'iridium 192, du césium 137 ou du cobalt 60. Exemple : la densité optique d'un film non exposé (bruit de fond) est de 0,20 , si ce dosimètre a été exposé à une dose de $0,20 \mathrm{mSv}$ (seuil de mesure habituel) aux rayons $\mathrm{X}$ de $100 \mathrm{kV}$ (énergie moyenne $40 \mathrm{keV}$ ), la densité optique sera de 0,60 , c'est-à dire une densité nette de 0,40 qui est très facilement mesurable avec une grande précision. Si ce dosimètre a été exposé à cette même dose de $0,20 \mathrm{mSv}$ avec des photons du cobalt $60(1130 \mathrm{keV})$, la densité optique sera de 0,25 soit une densité nette de 0,05 , ce qui pour un bruit de fond de 0,20 est très faible et peut être sujet à des erreurs de mesure. En effet, le densitomètre peut légèrement dériver ou le dosimètre qui a cheminé "aux quatre coins de l'hexagone » peut avoir pris une légère dose due à l'environnement. Autant il est simple de « tenir » un seuil de mesure de $0,20 \mathrm{mSv}$ en rayons $\mathrm{X}$ de basse énergie et il est même possible de prendre un seuil de $0,10 \mathrm{mSv}$, autant à des énergies supérieures à $400 \mathrm{keV}$, il est pratiquement impossible de descendre à moins de $0,20 \mathrm{mSv}$ avec une précision correcte.

Les difficultés d'automatisation: le traitement des dosimètres photographiques se prête mal à l'automatisation car le support est fragile et il nécessite une main-d'œuvre importante. De plus il faut examiner les dosimètres un par un car la lecture systématique en un point ou deux du dosimètre peut entraîner des erreurs en cas d'inhomogénéité manifeste (figure 2.7). Il s'agit d'un travail plutôt artisanal qui n'est plus très adapté aux concepts actuels. 
L'utilisation unique du dosimètre : le fait de garder le dosimètre après traitement est un avantage comme indiqué plus haut, mais c'est aussi un inconvénient car il faut renouveler les dosimètres tous les mois et procéder à de nombreuses vérifications et calibrations en fonction des lots de films, les réponses étant différentes d'un lot de fabrication à un autre.

\subsection{La dosimétrie par radio-thermo-luminescence}

Les dosimètres thermo-luminescents, appelés couramment TLD (Thermo Luminescent Dosemeter) sont constitués de fluorure de lithium (LiF), sulfate de calcium $\left(\mathrm{CaF}_{2}\right)$ ou borate de lithium $\left(\mathrm{Li}_{2} \mathrm{~B}_{4} \mathrm{O}_{7}\right)$ utilisés sous forme de poudre puis de pastilles frittées.

Sous l'effet des rayonnements, ces substances subissent des modifications de leur structure interne (figure 2.8). Si on les soumet à un chauffage uniforme, ils retournent à l'état initial en émettant une lumière dont la quantité est proportionnelle à la dose de rayonnements à laquelle ils ont été soumis.

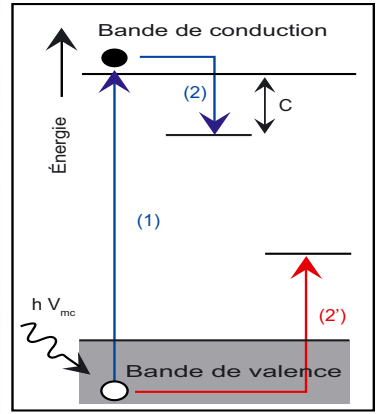

Irradiation

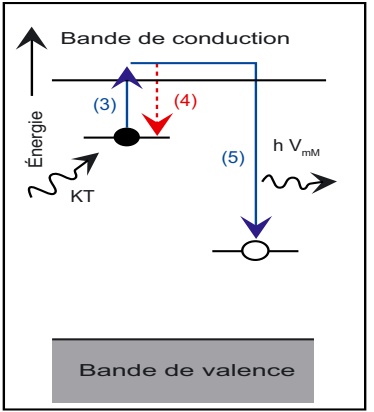

Thermostimulation

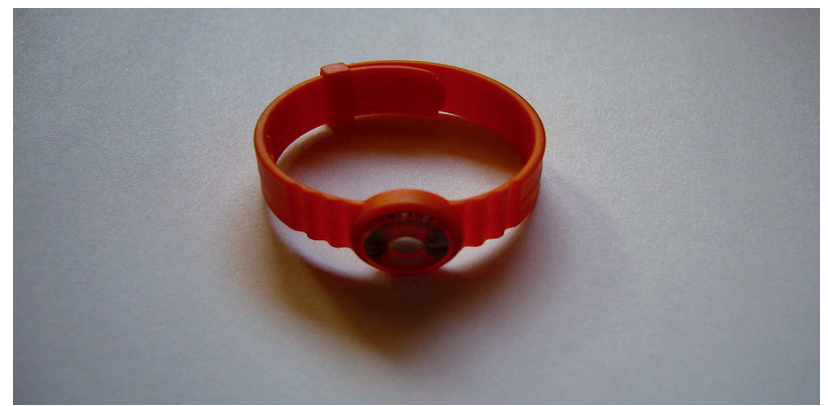

Figure 2.8 - Schéma de principe de la thermoluminescence et dosimètre thermo-luminescent TLD (bague IRSN). 
Dès les années 1960 Gui Portal (voir chapitre 8) a été, au CEA, le défenseur et promoteur de cette technique qui a été adoptée dans d'autres pays comme dosimètre pour la surveillance individuelle des travailleurs.

\section{Dosimétrie individuelle}

Pour utiliser le dosimètre TLD pour la surveillance dosimétrique individuelle, il faut disposer d'un badge comportant au moins quatre pastilles, en général une sans filtration et les trois autres couvertes par des écrans de cuivre, d'étain et de plomb de façon à évaluer l'énergie du rayonnement en cause. Il existe des modèles sous forme de plaquette rectangulaire comportant quatre pastilles réparties aux quatre angles, cette plaquette de la taille d'un dosimètre photographique est placée, dans un étui porte-écrans (Harshaw). D'autres se présentent sous forme d'une plaquette sur laquelle sont alignées quatre pastilles nues et couvertes d'écrans (Alnor, Panasonic).

En France la réglementation à imposé jusqu'en 1999, la dosimétrie photographique comme dosimétrie individuelle de référence (voir chapitre 4) mais la dosimétrie par thermoluminescence a été largement utilisée dans des domaines complémentaires :

La dosimétrie individuelle des extrémités sous forme de bagues munies de pastilles TLD très utile par exemple en médecine nucléaire pour mesurer la dose au niveau des doigts au contact de la seringue lors de l'injection de produits radioactifs au patient. Ce type de mesure est dans ce cas bien plus représentatif que la mesure sur un dosimètre porté au niveau du poignet.

Études de poste. Elles consistent à faire de nombreuses mesures dans des conditions de travail différentes. Ces mesures se font sur les personnels exposés (mains, front, gonades) et à des endroits stratégiques du local dans des conditions d'exposition représentatives des pratiques habituelles à ce poste de travail. Ces études permettent d'optimiser les doses des travailleurs par la bonne connaissance des paramètres d'exposition.

Reconstitution d'incidents. Il est possible d'équiper un fantôme anthropomorphe de multiples dosimètres (figure 2.9) et après avoir irradié ce fantôme dans les conditions de l'incident, la mesure de tous ces dosimètres permet d'analyser la répartition des doses dans l'individu exposé.

Dosimétrie des patients. La pastille thermo-luminescente peut être facilement utilisée conditionnée dans un petit sachet en vinyle qui prend peu de place et est transparent aux rayonnements X. Il suffit de placer sur la peau du patient une ou plusieurs pastilles ainsi conditionnées pour mesurer la dose à l'entrée du faisceau. 

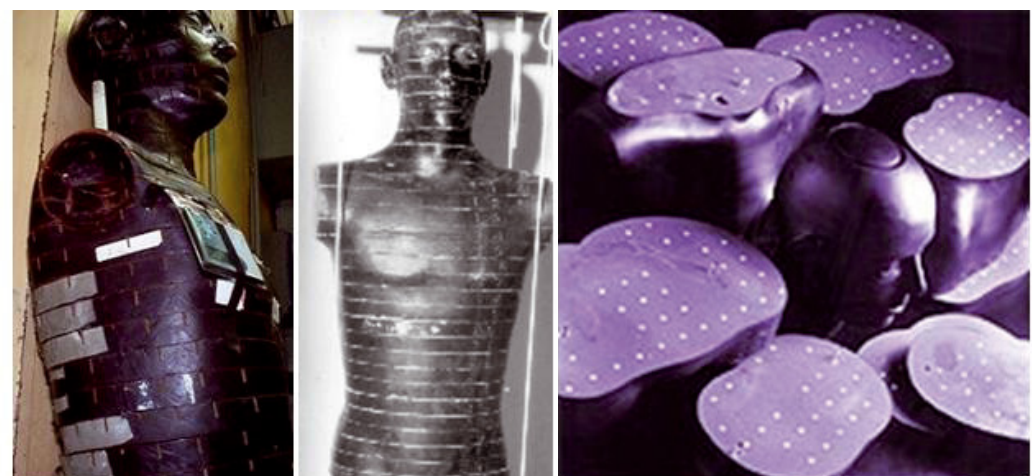

Figure 2.9- Reconstitution dosimétrique sur fantôme (IRSN). À gauche, figurent les dosimètres placés à l'extérieur du fantôme. À droite, figurent les emplacements des dosimètres sur chaque tranche du fantôme.

Ce type de mesures peut être effectué en radiodiagnostic pour vérifier le bon fonctionnement de l'appareil de radiologie et en radiothérapie pour contrôler les doses prescrites pour le traitement.

Dosimétrie de l'environnement. Compte tenu du seuil de mesure très bas, le dosimètre thermo-luminescent a été et est toujours largement utilisé pour les mesures du rayonnement naturel. En général, on le place dans un petit boitier pour des périodes de trois à six mois.

\subsubsection{Avantages et inconvénients du dosimètre thermo-luminescent (TLD)}

Du point de vue des qualités dosimétriques, le dosimètre thermo-luminescent est plus performant que le dosimètre photographique en termes de réponse en énergie et de sensibilité aux faibles doses, mais pour une utilisation à grande échelle dans des milieux très diversifiés, c'est un peu plus compliqué.

\section{Principaux avantages}

La qualité dosimétrique : le dosimètre lui-même a une réponse en énergie meilleure que celle du dosimètre photographique et elle peut être encore améliorée par l'adjonction d'écrans métalliques de natures différentes pour évaluer l'énergie des rayonnements. Il y a cependant des difficultés de mesure pour les faibles énergies, en particulier le sulfate de calcium a tendance à surestimer.

La sensibilité : il est possible de mesurer sans difficulté des doses de l'ordre de $0,05 \mathrm{mSv}$ quelle que soit l'énergie du rayonnement en cause. 
La possibilité de réutiliser le dosimètre après la mesure : une fois que le dosimètre revient à son état initial et il est prêt à être réutilisé. Ainsi un même dosimètre peut être attribué à une même personne et être utilisé plusieurs fois. Cette possibilité est intéressante surtout du point de vue financier, mais au bout d'un certain nombre d'utilisations la qualité dosimétrique se détériore et il ne peut être utilisé qu'un nombre de fois limité à une vingtaine.

La possibilité de faire des mesures ponctuelles : cette possibilité est importante pour des études dosimétriques sur patients ou sur fantômes comme indiqué sur la figure 2.9.

La possibilité d'automatisation : la lecture des badges peut se faire de façon automatique sur des chaines de mesure.

La fiabilité d'une technique éprouvée : ces dosimètres sont utilisés dans le monde entier depuis un demi-siècle dans différents domaines de la radioprotection.

\section{Principaux inconvénients}

Le coût : le dosimètre lui-même est beaucoup plus cher qu'un dosimètre photographique même si on peut l'utiliser plusieurs fois et, contrairement au film dosimétrique que l'on peut adresser seul à l'utilisateur, à charge de celui-ci de le placer dans un badge qu'il garde en permanence. Le dosimètre thermoluminescent sous forme de pastille doit être inclus dans un badge et il en faut au moins trois ou quatre pour tenir compte de l'énergie des rayonnements. Le nombre de dosimètres par badge et le poids du badge font que le dosimètre est plus coûteux de même que les frais de transport. Pour toutes ces raisons, ce dosimètre est plus rentable lorsqu'il est traité par un laboratoire présent dans les installations où sont mises en œuvre les sources de rayonnement.

L'impossibilité de relecture : une fois lu le dosimètre ne peut pas être relu pour vérification ou expertise, on peut simplement garder en mémoire la forme du spectre lumineux émis lors du chauffage. C'est la raison pour laquelle il vaut mieux avoir au moins deux dosimètres à chaque point de mesure.

\subsection{La dosimétrie par radio-photoluminescence (RPL)}

Cette technique est également basée sur le phénomène de luminescence provoqué par une stimulation physique d'un matériau qui a été soumis à une exposition aux rayonnements ionisants $X, \gamma$ ou $\beta$.

Le matériau utilisé est du verre dopé à l'argent phosphatique et l'agent physique est un faisceau de rayons ultraviolets. 
Malgré de bonnes qualités, il avait un défaut pour mesurer à grande échelle des doses de radioprotection susceptibles d'être reçues par des personnels exposés à des doses de l'ordre du millisievert. Ce défaut était la présence d'une pré-dose ou bruit de fond de l'ordre de grandeur des doses susceptibles d'être reçues $(0,2 \mathrm{mSv}$ environ).

Il a donc été essentiellement utilisé au CEA à des applications d'expertise d'environnement ou d'ambiance. Des années plus tard, un traitement nouveau a permis de rendre le dosimètre RPL compétitif pour des laboratoires généralistes de dosimétrie individuelle. C'est cette technique qui a été retenue par l'IRSN pour remplacer la dosimétrie photographique à partir de 2006, le basculement total sur la RPL ayant eu lieu en 2008 (figure 2.10).

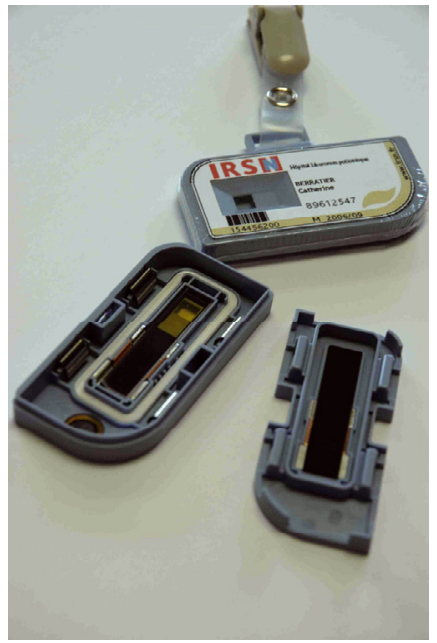

Figure 2.10 - Le dosimètre RPL de l'IRSN.

\section{Avantages et inconvénients du dosimètre par photoluminescence (RPL)}

Il est un peu tôt pour faire un état argumenté des avantages et des inconvénients de cette technique avec un recul suffisant, mais on peut toujours évoquer les avantages présents qui ont conduit au choix de l'IRSN qui dispose du laboratoire traitant le plus grand nombre de dosimètres en France.

\section{Principaux avantages}

L'excellente réponse en énergie : les tests de réponse en énergie montrent une bonne stabilité entre $30 \mathrm{keV}$ et $2 \mathrm{MeV}$ pour les $\mathrm{X}, \beta$ et $\gamma$. 
La possibilité d'obtenir des images : il est possible d'observer des différences de noircissement sous les écrans inclus dans le badge et la présence éventuelle d'un objet métallique interposé entre la source de rayonnements ionisants. La qualité de ces images n'est pas équivalente à celle du film mais c'est un atout intéressant (figure 2.11).

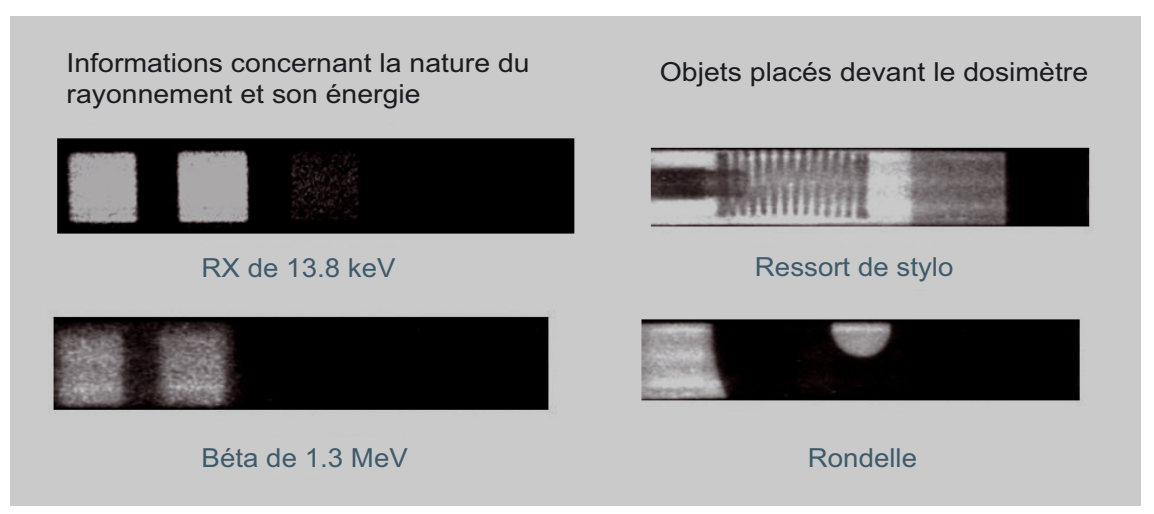

Figure 2.11 - Images obtenues sur des dosimètres RPL.

La possibilité de relecture : l'image enregistrée à la lecture du dosimètre est conservée et permet une nouvelle interprétation en cas de doute.

La possibilité de réutiliser le dosimètre : c'est un avantage comme pour le dosimètre thermoluminescent.

\section{Principaux inconvénients}

Il est difficile de trouver des inconvénients majeurs pour cette technique qui n'est utilisée à grande échelle en France que depuis 2008.

\subsection{La dosimétrie par luminescence optiquement stimulée (OSL)}

Le matériau utilisé dans ce dosimètre est de l'alumine $\left(\mathrm{Al}_{2} \mathrm{O}_{3}\right)$ et la luminescence est provoquée par exposition à un faisceau laser. La société Landauer a proposé à la fin des années 1990 à l'OPRI un dosimètre appelé « Luxel » qui était constitué d'un carré d'alumine de $1 \mathrm{~cm}$ de côté et placé dans un support de forme hexagonale très séduisant, léger et esthétique (figure 2.12).

Bien qu'intéressé dans la perspective de la disparition du dosimètre photographique, l'OPRI a repoussé cette offre pour des raisons techniques et surtout administratives. 


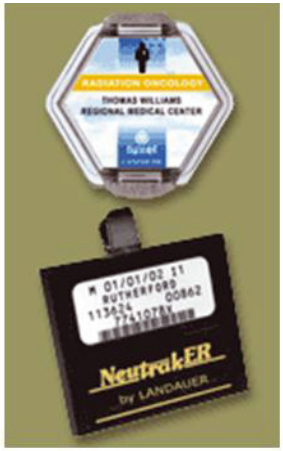

Figure 2.12 - Dosimètre OSL Landauer de type Luxel.

En 1999 la société Landauer s'est associée au LCIE pour créer l'entité LCIE Landauer et commercialiser un dosimètre OSL sous forme de pastilles d'alumine placées en ligne comme sur un dosimètre TLD.

Dès l'année 2000, le LCIE a abandonné le dosimètre photographique et a fourni à ses abonnés le nouveau dosimètre OSL au titre de LCIE Landauer et a assuré la fourniture et le traitement de ce type de dosimètre à EDF pour la surveillance dosimétrique de ses agents statutaires (voir chapitre 10). Cette technique est également utilisée au SPRA (voir chapitre 9).

Avantages et inconvénients du dosimètre à luminescence optiquement stimulée (OSL)

Il est un peu tôt pour tirer tous les enseignements de cette technique qui a priori présente également des avantages surtout en termes de sensibilité aux faibles doses.

\section{Principaux avantages}

Possibilité d'image : sous la forme « luxel » une grille métallique couvrant une partie du détecteur permet de distinguer une exposition unique d'une exposition chronique, mais il n'y a pas d'image au sens strict du terme.

Poids faible : le très faible poids permet de limiter les frais de transport entre le laboratoire et les abonnés.

\section{Principaux inconvénients}

Comme pour la RPL, il est prématuré de faire un état objectif des défauts de cette technique. 


\subsection{La dosimétrie par chambre d'ionisation}

Il s'agit d'une modernisation du concept de stylo-dosimètre basé sur l'ionisation de l'air avec la technologie des mémoires actives. Ce dosimètre est proposé depuis l'année 2000 par la société RADOS (figure 2.13) et il peut être utilisé en dosimétrie passive ou différée mais aussi en dosimétrie active si l'on place le dosimètre dans un lecteur portable.
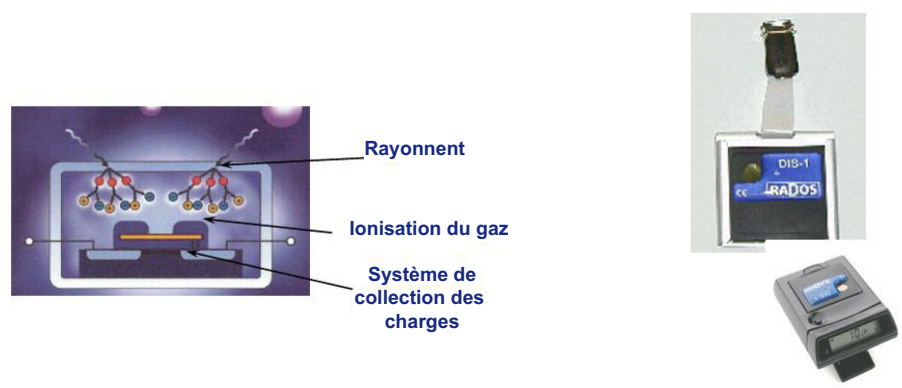

Figure 2.13 - Dosimètre RADOS

Le laboratoire du Centre européen de recherche nucléaire (CERN) l'utilise pour une partie de ses chercheurs. Du point de vue strictement dosimétrique, il est excellent par sa réponse en énergie et sa sensibilité mais il est assez peu utilisé en raison de son coût notamment.

\section{Avantages et inconvénients du dosimètre par chambre d'ionisation}

Il n'est pas utilisé à très grande échelle, ce qui rend difficile une comparaison avec les techniques décrites ci-dessus.

\section{Principaux avantages}

Qualités dosimétriques intrinsèques : c'est un dosimètre au sens strict du terme qui présente les qualités de réponse en énergie car il est constitué de matériaux équivalent-tissus, c'est-à-dire dont les teneurs en carbone, hydrogène, oxygène et azote sont proches de celles des tissus biologiques, et il est basé sur l'ionisation des gaz qui est le phénomène physique le plus utilisé en dosimétrie des rayonnements ionisants depuis l'origine.

Possibilité de dosimétrie active : la chambre d'ionisation, sous forme d'un carré de $4 \times 4 \mathrm{~cm}$ peut être placée dans un boitier portable et servir comme dosimètre actif avec lecture en temps réel des doses reçues par le porteur. 


\section{Principaux inconvénients}

Le coût : supérieur aux autres techniques.

La fragilité : il doit être bien protégé pendant le transport car, constitué de matériaux plastiques équivalent tissus, il peut se casser en cas de choc.

\subsection{Cas particulier de la dosimétrie des neutrons}

Pour mesurer les doses de neutrons reçues par les travailleurs, dans des installations de la filière nucléaire, on se base sur des mesures d'ambiance avec des radiamètres spécifiques à la mesure des neutrons rapides ou des systèmes plus sophistiqués comme des spectromètres à sphères de Bonner (Serre, 2010) (figure 2.14). Ces spectromètres sont constitués d'un jeu de 6 à 8 sphères en polyéthylène de diamètres différents contenant en leur centre un détecteur de neutrons thermiques produits par le ralentissement des neutrons rapides dans chaque sphère. Ces mesures dans les différentes sphères permettent de déterminer les fluences de neutrons par intervalle d'énergie et d'en déduire par calcul les débits de doses auxquels les travailleurs sont susceptibles d'être exposés. À partir du temps de présence et des conditions de travail, les doses des agents concernés sont évaluées.

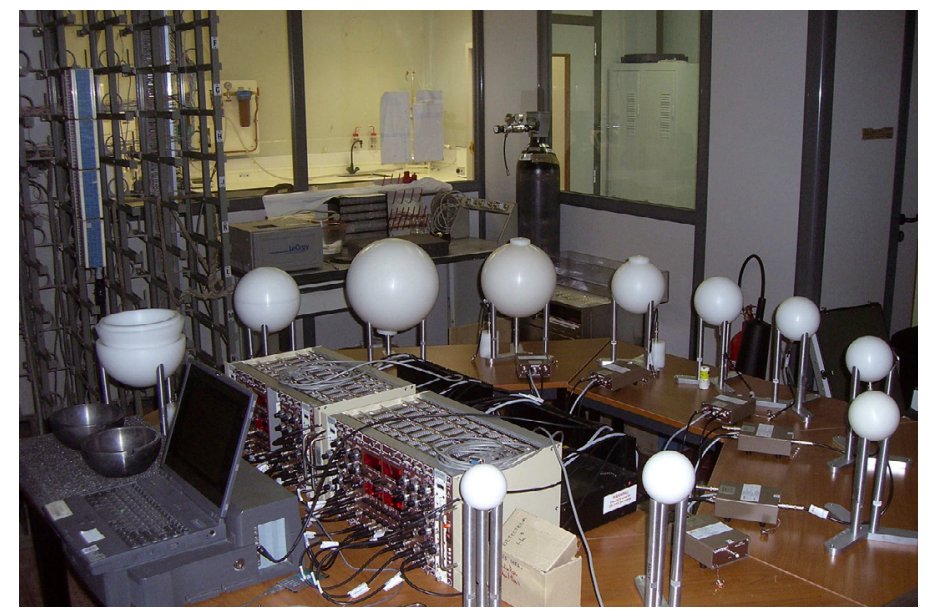

Figure 2.14 - Spectromètre à sphères de Bonner.

En ce qui concerne la dosimétrie individuelle, pour les neutrons thermiques la méthode la plus courante consiste à placer un écran de cadmium dans un dosimètre photon classique et mesurer les photons $\gamma$ issus de la réaction $n, \gamma$ des neutrons thermiques produite dans cet écran. 
Pour les neutrons rapides, le principe est d'utiliser des matières dans lesquelles les neutrons laissent des traces dont le comptage permet d'évaluer la fluence de neutrons et la dose.

Dosimètre à bulles : la matière sensible est une graisse compressée dans un dispositif semblable à un stylo dosimètre (figure 2.15). L'impact des neutrons sur cette graisse provoque l'apparition de «bulles » dont le nombre est proportionnel aux flux de neutrons. Ce dosimètre peut s'utiliser comme un dosimètre opérationnel par le porteur qui compte à l'œil nu le nombre de bulles formées et comme dosimètre passif lorsque le nombre de bulles est trop important pour être compté simplement. Dans ce cas, le dosimètre est placé dans un lecteur avec un faisceau lumineux dont on mesure l'absorption dans la graisse et qui est liée aux nombres de bulles.

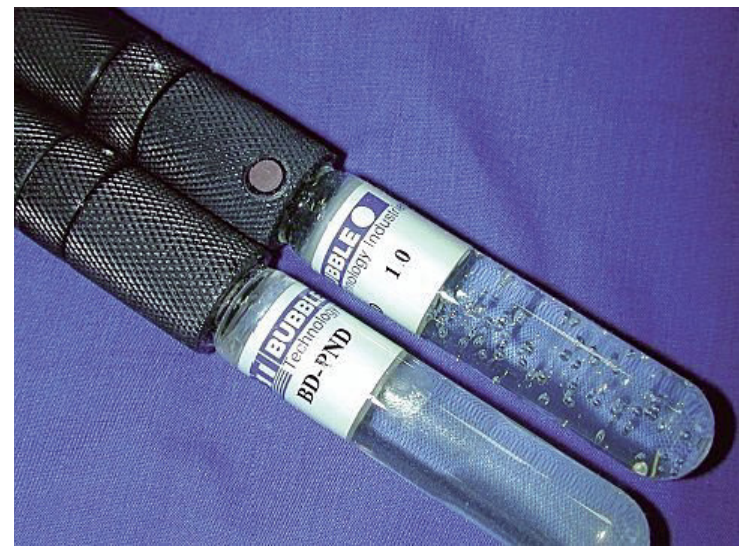

Figure 2.15 - Dosimètres à bulles de la société Bubble Technology Industrie, celui de dessus a été exposé à des neutrons rapides (présence de bulles).

Dosimètre photographique Kodak NTA : il s'agit d'une émulsion photographique particulière très lente qui nécessite un traitement chimique spécifique. Les protons mis en mouvement par les neutrons provoquent des traces dans l'émulsion qui sont révélées par le traitement photographique. Ces traces sont alors comptées avec un microscope et de ce comptage on déduit la dose due aux neutrons rapides. Cette technique a été utilisée au SCPRI et à l'OPRI et par les autres laboratoires jusqu'en 2000. Depuis, Kodak a arrêté la fabrication de ce type de dosimètre.

\section{Détecteurs de traces CR 39 et PN3}

L'impact sur le détecteur (polycarbonate) des protons mis en mouvement par les neutrons crée des traces qui sont révélées par un traitement à la soude et le 
nombre de ces traces comptées avec un dispositif automatique est proportionnel à la dose neutrons reçue. C'est le système adopté en 1998 à l'OPRI, mis en œuvre en 2000 et toujours utilisé à l'IRSN et dans la plupart des laboratoires.

\section{Dosimètres thermoluminescents}

Ce système est basé sur le principe de l'" albédo » qui consiste à mesurer l'exposition aux neutrons thermiques produits par le ralentissement des neutrons rapides dans le corps du porteur du dosimètre.

Le dosimètre comprend une pastille de fluorure de lithium enrichi en lithium 6 très sensible aux neutrons thermiques et une autre pastille de fluorure de lithium enrichi en lithium 7 insensible aux neutrons thermiques. La différence sur les deux lectures donne une indication sur l'exposition aux neutrons thermiques et par déduction la dose due aux neutrons rapides.

Pour être valable, cette technique nécessite des étalonnages en fonction des champs de rayonnements présents au poste de travail. C'est pour cette raison que cette technique a été utilisée essentiellement dans les laboratoires comme ceux de COGEMA (AREVA) qui sont présents sur le terrain et connaissent bien les postes de travail.

\section{RÉFÉRENCE}

Serre S. (2010) Développement d'un système de spectrométrie de neutrons étendu au domaine des hautes énergies, Thèse IRSN, Laboratoire de métrologie et de dosimétrie des neutrons (LMDN). 